\title{
Keyword Index Volume 34 (2014)
}

abdominal muscles

acquired brain injury

activity

adolescents

adults

aerobic exercises

age-related macular degeneration

Alzheimer's disease

animal models

anterior pituitary

aphasia

assessment

assistive technology

automobile driving

autonomic status

back muscles

balance disorders

balance

BDNF

behavior disorders

behavioral facilitation

bilateral vestibular loss

body weight support

body weight supported treadmill training

BoNT-A

Botulinum Neurotoxin A

botulinum toxin

brain damage

brain injuries

brain injury

brain morphometry

brain networks

brain plasticity

brain tumour

brain-computer interface (BCI)

burden

caregiver

cerebral palsy

cervical spinal cord injury

child abuse

coercion

cognition disorders

cognition

cognitive enhancement

cognitive impairment
323

$45,65,671$

297

147

463

209

587

101

101

625

499

409, 463

749

157

227

323

789

113, 193, 337, 343

209

709

637

799

323,731

287

185

185

515

147

157

$3,23,39,515,573,625,645,759$

81

479

81

499

355

313

313

515

473

373

671

613

$29,201,455$

101

531 cognitive performance

cognitive rehabilitation

cognitive training

cognitive-behavioral

communication

community integration

community re-entry

computerized dynamic posturography

constraint-induced movement therapy

context sensitive treatment

contracture

45

control

coping

corticospinal tract

critical illness polyneuropathy

culture change

culture

CVA

deficit awareness

depression

detraining

diffusion tensor imaging

disability

diurnal change

diversity

dizziness

DSM IV

DSM-5

dynamometry

$3,23,39,65,81,87$

81

709

749

157

637

343

201

709

215

429

55

259

493

655

645

267

45

455

523

81,259

$313,455,531$

227

645

221

455

613

287

eccentric viewing training $\quad 587$

efficacy $\quad 515$

electric stimulation therapy 245

electroencephalography (EEG) 355

electromyography (EMG) 381

electromyography 277, 323

electromyography-triggered neuromuscular

stimulation

EMG

323

emotion recognition 23,39

endocrine $\quad 625$

$\begin{array}{ll}\text { equinus } & 215\end{array}$

eventrelated desynchronization (ERD) 355

executive function $\quad 55$

exercise rehabilitation $\quad 523$

$\begin{array}{lr}\text { expressive writing skill } & 29\end{array}$ 
eye condition

eye movements

family needs

fatigue

finger

function

functional assessment

functional independence measure

functional recovery

functioning

F-wave

gait analysis

gait speed

gait training

gait

gender

gleno humeral subluxation

hand function

hand rehabilitation

hand

heart rate variability

hemiplegia

hemisphere

high dose

home health care (HHC)

home-based rehabilitation

HPA axis

H-reflex

hypopituitarism

ICF

incomplete spinal cord injury

inpatient rehabilitation

insight

interdisciplinary

inter-limb learning transfer

intervention

ischemic stroke

keyboard playing

kinematics

language impairment

language

Latin America

leisure engagement

life satisfaction

locomotor training

lokomat

longitudinal studies

low back pain

lower extremity
725 M wave

magnetic resonance imaging

$$
\text { mental health }
$$

167,671

167 mental practice

meta-analysis

microsoft kinect

MIDI

147

mild head injury

741

391, 493

military training

473

773

mirror therapy

235

337

725 Modified Tardieu Scale $\quad 177$

mothers 373

323,731

motion analysis

259

355

121

447

$185,193,253,259,267,323,731$

motor cortex

355,725

motor imagery

motor outcomes

113

297

429,773

motor

129

409

147

429

227

mTBI

709

$\begin{array}{lr}\text { multidisciplinary approach } & 809 \\ & 655\end{array}$

235, 447

multidisciplinary

749

277

multiple disabilities

731

515

multiple sclerosis

523

531

muscle strength

287,523

625

177

nearvision symptoms

129

neuroendocrine injury $\quad 625$

625

neuroimaging

479

neuroplasticity

129

297

neuropsychological assessment

201

287

neurorehabilitation

$625,709,809$

neurosensory

809

3

neurosurgery

499

655

neurotrauma

637

297

637, 709 non-invasive brain stimulation

437, 789

23,39

147

nonverbal communication

587

129

129

129

499

oculomotor deficiency

oculomotor learning

493, 759

463, 499

culomotor rehabilitation

473

749

313

Pakistan

373

541,557

parenting

541, 557

287, 447

Parkinson's disease

297

157

participation

287

113

peak torque

523
731

pelvis 
perceptive rehabilitation

permanent post concussion symptoms

personality change

pharmacology

philosophy of mind

phosphodiesterase inhibition

physical exercise

physiotherapy

pilates

positive behavior support

positive psychology

positive psychotherapy inventory

post-acute recovery

post-acute rehabilitation

post-acute

post-coma persons with consciousness

postschool outcomes

post-stroke spasticity

posture

prevalence

progressive resistance training

psychoeducational

psychoneuroimmunology

PTSD

quality of life

reading dysfunction

rehabilitation team

rehabilitation

reliability

resilience

restraint

rhythmic auditory stimulation

role release

school psychologist

school-to-work transition

scope of practice

seclusion

secondary prevention

selectivity

self concept

self-efficacy

self-rated health
113 sensory conflicts

343

741 sensory organization test 343

601 severe $\quad 773$

637 shoulder 245

601 skilled nursing facility (SNF) 531

101 social isolation 625

401 spasticity $177,185,515$

409 stimulus artifact suppression $\quad 381$

337 strength 337

709 stroke rehabilitation 235

573 stroke 55, 87, 177, 193, 201, 209, 221, 245,

253, 799

573

671

193

655

479

365

655

671

401

429

3

313

221
253, 259, 267, 297, 391, 409, $429,437,455,493,773$

survivors

455

systematic review

499

TBI caregivers

167

TBI

$29,479,625$

121

team models $\quad 655$

test validity 463

$\begin{array}{ll}\text { tetraplegia } & 473\end{array}$

TIA 401

toe walking 215

tracheostomy $\quad 809$

transcranial stimulation $\quad 437$

transdisciplinary $\quad 655$

trauma 671

traumatic brain injury $\quad 87,129,365,373,601,613,709$

traumatic 157

treadmill 323,731

$\begin{array}{ll}\text { treatment } & 121,671\end{array}$

trunk midline 113

$\begin{array}{ll}\text { trunk } & 731\end{array}$

$\begin{array}{ll}\text { upper extremity 201, } 235 & \end{array}$

validity

177,799

343

437

759

walking

$323,493,731$

wii balance board

789

437

$\begin{array}{lr}\text { Wisconsin Gait Scale } & 253 \\ \text { writing disorder } & 29\end{array}$ 